The symmetry of the perturbing crystal field acting on the tetrahedral $\mathrm{BH}_{4}{ }^{-}$is thus $C_{8}$. Under the action of a perturbing field of symmetry $C_{8}$, the vibrational levels of the tetrahediral ion transform as follows :

$$
\begin{aligned}
& T_{d} \quad C^{s} \\
& \stackrel{A^{d} \rightarrow A^{\prime}}{ } \\
& E \succeq A^{A^{\prime \prime}} \\
& \underset{F_{2} \rightarrow A^{\prime}}{A^{\prime \prime}} \\
& \searrow A^{\prime}
\end{aligned}
$$

that is, the degeneracy of all the levels is removed and all the levels are infra-red active (only the $F_{2}$ levels are infra-red active under $T_{d}$ ).

This means that all four tetrahedral fundamentals should be observable in the infra-rod absorption spectrum, as well as all the overtones and combination bands. In addition, all the degenerate bands should show fine structure caused by the rernoval of all the degeneracy by the porturbing erystal field.

The splitting of the degenerate bands would probably be obscured by the broadness of the bands at room temperature, but $\nu_{1}$ and $v_{2}$ should be observable. Unfortunately, the band due to the $\nu_{1}$ vibration lies among $v_{3}$ and its satellite bands (at about 2,300 cm..$^{-1}$ ) and could easily be obscured by these.

From the spectrum published by Price $^{1}$ it can be deduced that $v_{2}$ should occur around $1,280 \mathrm{~cm} \cdot{ }^{-1}$ (from the frequencies of $v_{4}$ and $v_{2}+v_{4}$ ).

The anomalous band reported by Price $^{1}$ at about $1,250 \mathrm{~cm} .^{-1}$ in the spectrum of lithium borohydride can thus probably be assigned to the $v_{2}$ vibration of the $\mathrm{BH}_{4}$ - ion in the lithium borohydride lattice. This band is normally inactive in the infra-red, but occurs here because of the 'reduction' of the symmetry of the ion by the crystalline field, which is of the type $C_{s}$.

$$
\text { C. J. H. Schutte }
$$

Chemical Physics Group of the National Physical and Chemical Research Laboratories,

Council for Scientific and Industrial Research, Pretoria.

${ }^{2}$ Price, W. C., J. Chem. Phys., 17, 1044 (1949).

2 Schutte, C. J. H., Spectrochim. Acta, 16, 1054 (1960).

'Waddington, T., J. Chem. Soc., 4783 (1958).

- Schutte, C. J. H. thesis, University of Amsterdam (1960).

${ }^{5}$ Harris,P. M., and Meibohm, E. P., J.Amer. Chem. Soc., 69, 1231 (1947).

'Halford, R. S., J. Chem. Phys., 14, 8 (1946).

\section{Isolation of D-Glycero-D-galacto-heptitol from the Wound Exudate of Avocado Trees}

D-GLYCERO-D-GALACTO-HEPTITOL has been isolated from the semi-crystalline exudate of wounds in the bark of Persea americana Mill.

The crude material was a pale brown solid which did not reduce Fehling's solution, and gave evidence of only one major component on paper chromatograms. Two recrystallizations of this solid from aqueous methanol afforded a colourless product in the form of needles which had a melting-point of 183-185 ${ }^{\circ}$, no dopression on admixture with authentic perseitol.

A portion of the crude exudate was acetylated with pyridine and acetic anhydride to give a crystalline acetate, which after recrystallization from aqueous et. $\mathrm{k}$ anol had a melting-point of $119-120 \cdot 5^{\circ}$, no depression on admixture with authentic perseitol heptace. tate. This derivative also had $[\alpha]_{D}^{230}-14^{\circ}$ (about $2 \cdot 02$ in chloroform); literature value $\theta^{1}[\alpha]_{D}^{200}-13 \cdot 3^{\circ}$ (chloroform).

A further portion of the crude material was treated with benzaldehyde and acid according to Hudson et $a l .{ }^{2}$. From this reaction mixture a crystalline derivative was isolated, which after one recrystallization from pyridine-othanol had a melting-point of $276-278^{\circ}$ and $[\alpha]_{D}^{20^{\circ}}-62^{\circ}$ (about 1.95 in pyridine). Hudson et al. ${ }^{2}$ give values of $277 \pm 2^{\circ}$ for the melting. point and $[\alpha]_{D}^{200}-58.1^{\circ}$ (about 0.9 in pyridine) for I,3 : 5,7-di-O-benzylidene-D-glycero-D-galacto-heptitol.

From these results we have concluded that the chief constituent of Persea americana Mill wound exudate is D-glycero-D-galacto-heptitol (perseitol), a compound known to be present in the fruit ${ }^{3}$ and leaves ${ }^{4}$ of this tree.

Thanks are due to Drs. L. W. Klotz and G. A. Zentmyer of the Citrus Experiment Station, Riverside, California, who supplied the exudate described here.

\section{J. K. N. JONES}

R. A. WALL

Gordon Hall,

Queen's University,

Kingston, Ontario.

${ }^{1}$ Maquenne, L., C.R. Acad. Sci., Paris, 107, 583 (1888).

${ }^{2}$ Ness, A. T., Hann, R. M., and Hudson, C. S., J. Amer. Chem. Soc., 70,767 (1948)

${ }^{3}$ Montgomery, E. M., and Hudson, C. S., J. Amer. Chem. Soc., 61, 1657 (1939).

4 Nordal, A., and Benson, A. A., J. Amer. Chem. Soc., 76, 5054 (1954).

\section{Monosaccharide Sequence in Pneumococcus Type XIV Polysaccharide}

Prevrous work ${ }^{1}$ has established that in Pneumococcus type XIV polysaccharide there are two nonreducing end groups of D-galactopyranose, one D-galactopyranose residue linked $1: 3$, one D-glucose residue linked $1: 4$ and two $1: 4: 6$-linked 2-acetamido 2-deoxy $D$-glucose residues for every six monosaccharide units. Although the majority of these linkages are $\beta$-, some $\alpha$-D-galactose units may be present. The two major disaccharides left after treatment of the type XIV polysaccharide with $N$ hydrochloric acid at $100^{\circ}$ for $2 \frac{1}{2} \mathrm{hr}$. were 4-O- $\beta-\mathrm{D}$ glucosyl 2-acetamido 2-deoxy $D$-glucose and 3-O- $\beta$ (2-acetamido 2-deoxy)-D-glucosyl D-galactose. Less drastic hydrolysis has enabled other disaccharides to be isolated and the structure of these is the subject of the present communication.

The polysaccharide $(3.606 \mathrm{gm}$.$) was hydrolysed$ with $2 N$ sulphuric acid $\left(430 \mathrm{ml}\right.$.) at $85-90^{\circ} \mathrm{C}$. for $21 \mathrm{hr}$. After neutralization with barium carbonate, the hydrolysate was passed through 'Amberlite $I R$ ' $120\left(\mathrm{H}^{+}\right)$, which was eluted with water to yield neutral sugars ( $1.8 \mathrm{gm}$.) and with $2 \mathrm{~N}$ sulphuric acid to remove basic sugars $(1.0 \mathrm{gm}$.$) . Fractionation of the neutral$ sugars on a charcoal-'Celite' column" gave a $\mathrm{D}$ galactose-D-glucose mixture (about 1.4 gm.), Nacetylglucosamine (40 mgm.), disaccharides I and II (4 mgm.), III (11 mgm.), V (30 mgm.), VI (3 mgm.), trisaccharides IV and V (30 mgm.), as well as trisaccharide VI and another oligosaccharide (30 mgm.). 